\title{
I solation and partial characterization of lytic phage against Salmonella Abortusequi
}

Harshit Verma, Divya Pramod, Mohd. Abbas, Awadhesh Prajapati, Durairajan Ramchandra, Mayank Rawat

Division of Biological Standardization

Indian Veterinary Research Institute, Izatnagar, India

Corresponding author: Mayank Rawat, email: mayankvet9@gmail.com

Received: 21-06-2012, Accepted: 07-07-2012, Published online: 06-12-2012

\section{How to cite this article:}

Verma H, Pramod D, Abbas M, Prajapati A, Ramchandra D and Rawat M (2013) I solation and partial characterization of lytic phage against Salmonella Abortusequi, Vet World 6(2): 72-75. doi: 10.5455/vetworld. 2013.72-75

\begin{abstract}
Aim: To use partially characterized phages for generation of phage lysates having potential to be developed as vaccines against Salmonella Abortusequi.

Material and Methods: Phage/s isolation from several sewage samples was attempted against Salmonella Abortusequi strains procured from the National Salmonella Reference Centre, IVRI using enrichment and agar overlay technique. A concentrated stock of the phage was prepared after its purification. The lytic range, morphology and DNA size were determined.
\end{abstract}

Results: Two lytic phages could be isolated and purified. The phage with wider lytic range was found to possess a hexagonal head with a very short tail. The DNA size was found to be $21 \mathrm{~kb}$.

Conclusion: The investigation reports first isolation of a lytic phage against Salmonella Abortusequi. The phage can be used in future for development of phage lysate based immune therapeutic agent against equine abortions.

Keywords: bacteriophage, lytic phage, Salmonella Abortusequi

\section{Introduction}

Equine genital tract infections due to Salmonella Abortusequi are a cause of major concern to the stud owners and breeders. These infections inflict severe economic losses by way of abortions, breeding inconsistencies and high cost of maintaining infertile mares over a long period of time. Prophylaxis and control of contagious equine abortion largely depends upon vaccination against the infection. But the options for the vaccines against $S$. Abortusequi are limited [1]. The public health risk varies by animal species, age group, husbandry practice and health status, and certain human subpopulations are at a heightened risk of infection due to biological or behavioral risk factors [2].

Currently available prophylactic compositions are poorly standardized inactivated, adjuvanted bacterial whole cell suspensions of one or more strains of the organism. These products induce a short-lived antibody mediated immune response without eliciting any cell mediated immunity, which is essential for intracellular pathogens such as $S$. Abortusequi [3]. Attempts to develop newer, improved vaccine compositions, specially the live attenuated vaccines have met with variable results and have not found merit to be developed into safe product to be used routinely in field.

The generally accepted explanation for poor responses of killed vaccines relates to the alteration of antigens during the process of chemical \& physical inactivation, or protein extraction. Parry [4] showed that live $E$. coli were superior to heat-killed or formalinized organisms at eliciting specific mucosal immunity. The reasons were thought to be alterations in the antigenic moieties by chemical or physical inactivation of bacteria. Similar observations have also been made by other researchers with other organisms $[5,6,7]$.

It is therefore, hypothesized that a method of inactivation or antigen extraction that does not or minimally alter the antigenic moieties of the organism is the key to developing an effective immunizing preparation capable of inducing both $\mathrm{CD} 4^{+}$and $\mathrm{CD} 8^{+} \mathrm{T}$ cell responses. A preparation containing complete range of structurally unaltered antigenic moieties of the bacterial cell is not only expected to mimic a nonreplicating live organism in the host and induce the desirable protective response at significantly lower doses, but also circumvent all other problems of killed, subunit or live attenuated vaccines.

In view of current understanding of the fundamental concepts of bacteriology and immunology, and the state of art of production of immunobiologicals against bacterial diseases, it has to be accepted that the development of an ideal immunizing agent against any bacterial or viral pathogen is a very difficult task. But there are options that have not been properly investigated till now and can be explored to improve upon the limitations of currently available preparations against all bacterial diseases including all Salmonelloses 
in general and, $S$. Abortusequi in particular. One of the most suitable and practicable method may be the use of lytic phages as inactivating agents, and employing "phage lysate bacterins" as immunizing agents.

Phage lysates comprise a means of effectively killing bacteria while minimally altering their antigenicity [8]. Phage lysate bacterin contains bacteriophage particles (primarily responsible for the first phase of protection mediated by phage itself) released by the burst, and bacterial antigenic components (associated the second phase of protective response mediated by antibodies or effecter cells) produced by the burst caused by lytic bacteriophage. Under this scenario, phage lysate bacterin against $S$. Abortusequi presents an opportunity to improve upon the currently available vaccines for control of contagious equine abortions.

The mandatory requirement for developing phagebased immunotherapeutic preparations is availability of consistently lytic phages against the target organism / organisms. So far there are no reports on isolation of lytic phages against $S$. Abortusequi from India and for that matter, from any other country in which equine abortions are prevalent. The present investigation was therefore undertaken to isolate and partially characterize consistently lytic phages against $S$. Abortusequi so that in future they can be used for development of phage lysate based immuno-therapeutic compositions against contagious equine abortions.

\section{Materials and Methods}

Bacterial strains: Three Salmonella Abortusequi reference strains E- 155, E-156, E-157 were procured from the National Salmonella Centre, IVRI, Izatnagar, and maintained at $4{ }^{\circ} \mathrm{C}$ on Nutrient Agar throughout the study.

Phage isolation and purification and preparation of concentrated stocks: Isolation of lytic phages against $S$. Abortusequi was attempted on sewage samples collected from several places such as animal farms having horses, institute experimental animal sheds, animal and human hospital drainages and, stagnant ponds by following the methodology of Atterburry [9]. Minor modifications with respect to the use of growth medium and diluents were made. The plaques obtained on agar overlay plates were subjected to purification by liquid culture and soft agar streak method [10]. Concentrated stocks of purified phages were prepared by agar wash method of Sambrook and Russell [10]. Briefly, About $2 \mathrm{ml}$ of SM diluent $(50 \mathrm{mM}$ Tris-Cl [pH 7.5], $0.1 \mathrm{M} \mathrm{NaCl}, 8 \mathrm{mM} \mathrm{MgSO}_{4} .7 \mathrm{H}_{2} \mathrm{O}, 0.01 \%$ Gelatin) was added to each of several agar overlay plates with plaques against $S$. Abortusequi, and kept for $6 \mathrm{hrs}$ at $4^{\circ} \mathrm{C}$. The fluid was collected, and the process was repeated once more. Both collections (about $4 \mathrm{ml}$ from each plate) were combined and filtered through 0.22 $\mu \mathrm{m}$ syringe filters, after centrifugation if necessary. Sterile filtrate constituting concentrated phage preparation was collected aseptically in sterile tubes of appropriate capacity. The preparations were once again tested for presence of phage against, respective indicator $S$. Abortusequi by agar overlay, pooled and stored at $4^{\circ} \mathrm{C}$.

Enumeration of phage in concentrated stocks: The phage count of the sterile stocks was determined by serial 10 fold dilution followed by plaque count on soft agar overlay.

Host range: For determination of lytic range of the phages, $3 \mu l$ amounts of test phages were spotted on spread lawn culture of $S$. Abortusequi 155, 156 and 157; Salmonella Gallinarum; Salmonella Pullorum; Pasteurella multocida B:2 $\left(\mathrm{P}_{52}\right)$ and Staphylococcus aureus. After the spots dried, the plates were incubated at $37^{\circ} \mathrm{C}$ for $24 \mathrm{~h}$ and observed for presence of clear lytic zones between the lawns of growth.

Phage morphology: Negative staining electron microscopy of phage was conducted for morphological characterization. Phage suspension (adjusted to $10^{8} \mathrm{pfu} / \mathrm{ml}$ ) was mixed with $2.5 \%$ of glutaraldehyde and kept for $1 \mathrm{hr}$ at 4?C. $25 \mu l$ of fixed phage was added to surface of grid and left for $2 \mathrm{~min}$. The phage was negatively stained with $0.5 \%$ uranyl acetate for $2 \mathrm{~min}$ and observed in electron microscope.

Phage DNA: Phage DNA was isolated by using Lambda DNA Extraction Kit (Qiagen, USA) according to the method recommended by the manufacturer. The quality and molecular size of the DNA was analysed by agarose gel electrophoresis in $0.7 \%$ horizontal slab submarine gel containing ethidium bromide $(10 \mathrm{mg} /$ $\mathrm{ml}$ ), using 1x TAE buffer following the method of Sambrook and Russell [10].

\section{Results}

I solation of lytic phage against $S$. Abortusequi: Several bacteria free filtrates (BFF) prepared and screened for detection of lytic activity against $S$. Abortusequi strains included for the study. All BFF showing lytic activity against any $S$. Abortusequi strain were selected for detection of phage by agar overlay. The BFFs showing presence of lytic phage as indicated by presence of plaque (Fig-1) were further processed for purification. Two phages with consistent lytic activity could be isolated during the study and were named as PSAE/1 and PSAE/2. The phage count of pooled concentrates of both was found to be in the range of $5 \times 10^{10} \mathrm{pfu} / \mathrm{ml}$.

Host range: PSAE/1 was found to be lytic against all $S$. Abortusequi strains included for the study whereas PSAE/2 was to lytic to only the indicator strain (E155) against which it was isolated. Both phages did not show any across- Salmonella biovar or across-genus lytic range. Being a phage with wider host range, PSAE/1 was selected for further characterizations.

Electron Microscopy of phage: The negative stain electron microscopy of SAE/1 revealed presence of a hexagonal head and a well marked short tail. (Fig-2). 


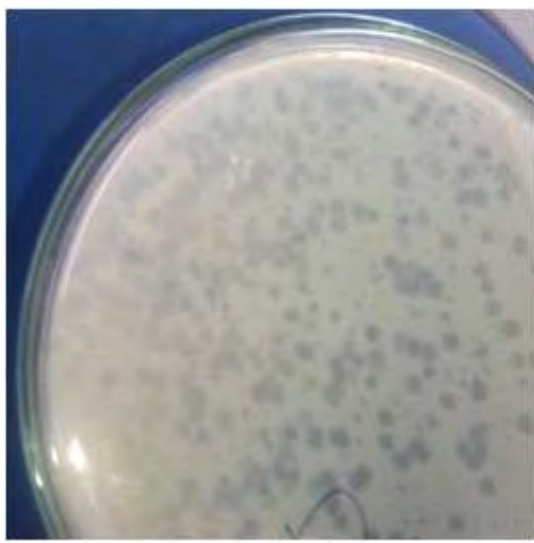

Figure-1. Agar overlay plate showing plaques

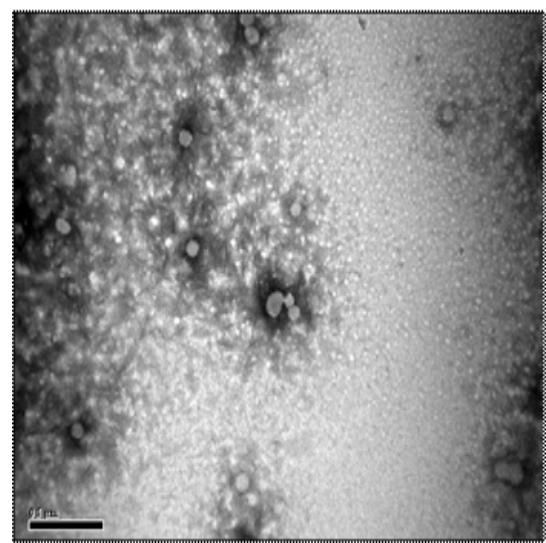

Figure-2. Electron micrograph of Salmonella phage

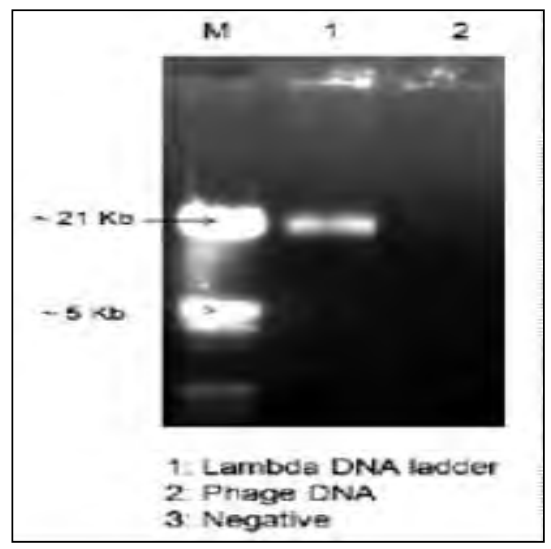

Figure-3. Phage DNA (21 kb)
Phage DNA: molecular size: The molecular size of the genomic DNA of SAE/1 was determined by $0.7 \%$ agarose gel electrophoresis using lambda mix genomic marker. The DNA of phage was found to be of about 21 kb(Fig-3).

\section{Discussion}

The initial requirement for developing phagebased immunotherapeutic preparations is the availability of consistently lytic phages against the target organism / organisms. As reported in previous studies the causes of abortion can change over time, as a reflection of improved diagnostic capability or because of background differences in at-risk equine populations [11]. So far there are no reports on isolation of lytic phages against $S$. Abortusequi from India. However, two consistently lytic phages could be isolated against two $S$. Abortusequi strains during the present investigation. The indicator strain of one phage (PSAE/2) was E155 and, for the other phage (PSAE/1), the BFF showed lysis of all the 3 strains. Both phages were isolated from experimental animal farm effluents of IVRI, Izatnagar. The present study is the first report on isolation of lytic phages against $S$. Abortusequi in India.

Presence of lytic phages against an organism is an indicator of presence of the organism itself in the environment. Bacteriophage can be isolated from most environments populated by bacteria and their numbers generally reflect the bacterial population [12]. Active vaccination during pregnancy may lead to abortion induced by heightened cell mediated immune response [13]. It appears from this experience that $S$. Abortusequi is present in large numbers in the environment. All phages can find some therapeutically important application at any time and efficacy of phages only when administered shortly after bacterial infection [14] are the most frequent criticisms of the clinical use of phages. Therefore, isolation of phages should be a continued activity. Just as for micro-organisms, a collection of large number of partially characterized, maintained and catalogued phages becomes an asset for the researchers and clinicians. Attempts for isolation of more phages against $S$. Abortusequi and other Salmonella serovars should be continued in future as well.

Determination of the lytic range of the phage is an important step that helps in selection of phage candidate / candidates for a specific purpose. Phages having lytic activity against a wide range of biotypes of the organism are preferred when they are intended to be used for therapeutic application. Phage designated as PSAE/1 was lytic on three of the 5 Salmonella strains whereas, PSAE-2 was found to lyse only on the indicator strain (the strain against which the phage was originally isolated) showing only a very narrow host range. The phage with a broader range of lytic activity (PSAE/1) was selected for further investigations.

The negatively stained preparations of PSAE- 1 in Electron Microscopy revealed presence of a phage with a short tail and icosahedral head (Figure: 2 ). Since the tailed phage comprises $96 \%$ of currently identified bacteriophages, out of which the Siphoviridae constitutes $62 \%$, Myoviridae $25 \%$ and Podoviridae approximately $14 \%$ [15], further studies are needed to assign specific taxonomic affiliation of the phage. Since this was not the objective of the investigation, further studies in this direction were not conducted. The DNA of PSAE-1 was purified. In agar gel electrophoresis the DNA appeared to be of $21 \mathrm{~kb}$ size (Figure 3). Bacteriophage genomes consist of either single or double stranded DNA or RNA with extremely variable size ranging from 4-725 kb [12], which may be circular or linear. Further studies on genome size, RE patterns will help to establish the taxonomic affiliation of PSAE-1.

Recent research activities on development phage based "immunotherapeutic" compositions have shown immense potential for their application as therapeutic agents and vaccines. An additional advantage of phagebased immunobiologicals is that in order to increase the range of their activity, cocktails of preparations can be generated without any extra effort. Cocktails of Salmonella phage lysates have been generated and found to protect birds against all serovars of the organism affecting poultry [8]. A phage-based 
"immunotherapeutic" system can also be generated against the problematic $S$. Abortusequi. It can be expected that systematic research efforts in this direction will yield quick results that can be integrated with their field application. The phage isolated during the investigation may be used for development of an effective immunobiological composition against Contagious Equine Abortions.

\section{Author's contributions}

MR designed the study and all the other authors performed research experiment. MR drafted and revised the manuscript. All authors read and approved the final manuscript.

\section{Acknowledgements}

Authors are thankful to Head, Division of Biological Standardization and Director, IVRI for providing laboratories facilities.

\section{Competing interests}

Authors declare that they have no competing interest.

\section{References}

1. NRC report, Annual Report, Hissar, India. Year 1987-88; 8990;91-92; 94-95; 95-96; 97-98; 98-99.

2. Hoelzer k., Andrea Isabel Moreno Switt, Wiedmann M. (2011) Animal contact as a source of human non-typhoidal salmonellosis. Veterinary Research. 42:34.

3. Titball R. (2008) Reduced secretion of Yop J by Yersinia limits in vivo cell death but enhances bacterial virulence. Infec. Diseases. 4(5):67.

4. Parry S.H., Allen W.D. and Porter P. (1997) Intestinal immune response to E. coli antigens in the germ-free chicken. Immunology. 32: 731-741.

5. Holt M.E., Enright M.R. and Alexander T.J. (1990) Immunization of pigs with killed cultures of Streptococcus suis type 2. Res.Vet. Sci., 48(1):23-27.

6. Melamed D. Leitner G. and Heller D.E. (1990) A vaccine against avian colibacillosis based on ultrasonic inactivation of Escherchi coli. Avian Diseases. 35:17-22.

7. Lauvau, G., Vijh, S., Kong, P., Horng, T., Kerksiek, K., Serbina, N., Tuma, R.A., and Pamer, E.G. (2001) Priming of memory but not effector CD8 T cells by a killed bacterial vaccine. Science 294, 1735-1739.

8. Pasterneck. (2007) Method for vaccination of poultry by using Bacteriophage lysate bacterin. US 2009/0297561 A1.

9. Atterbury R.J., Bergen Van M.A.P., Ortiz F., Lovell M.A., Harris J.A., Boer D.A., Wagenaar J.A., Allen V.M. and Barroe P.A. (2007) Bacteriophage therapy to reduce Salmonella colonization of broiler chicken. Applied and Environmental Microbiology. 4543-4549.

10. Sambrook J. and Russell D.W. (2001) Molecular cloning a laboratory manual. Cold spring harbour laboratory press, Cold spring Harbour, New York, $3^{\text {rd }}$ edition.

11. Butler C. (2011) Surveillance of viral, bacterial and other causes of equine abortion in the United Kingdom: 20062011. Equine Quarterly Disease Surveillance Report 7(1): 1-3.

12. Ackerman, H.W. (1998) Tailed bacteriophages: The caudovirales. Adv.Viru.Res., 51:135-201.

13. Negi V. D., Arvindhan G. Nagarajan, Dipshikha Chakravortty. (2010) A Safe Vaccine (DV-STM-07) against Salmonella Infection Prevents Abortion and Confers Protective Immunity to the Pregnant and New Born Mice. PLoS. 5(2): 1-10.

14. M. L. Marenzoni, M. L., Lepri E., Casagrande P.P., Bietta A., Coletti M., Timoney P. J., Passamonti F. (2012) Causes of equine abortion, stillbirth and neonatal death in central Italy. Veterinary Record. 170:262.

15. Sharp, R. J., and P. Hambleton. (2001) A coming of phage: Bacteriophages in biotechnology. J. Chem. Technol. Biotechnol. 76:661-699. 